\title{
Logic Board Device
}

National Cancer Institute

\section{Source}

National Cancer Institute. Logic Board Device. NCI Thesaurus. Code C50037.

The circuit board that contains most of the processor units. 